Received: 2015.03.16

Accepted: 2015.04 .01

Published: 2015.06.01

Authors' Contribution:
A Study Design
B Data Collection
C Statistical Analysis
D Data Interpretation
E Manuscript Preparation
F Literature Search
G Funds Collection

\title{
Diffuse Subarachnoid Hemorrhage Secondary to Cerebral Venous Sinus Thrombosis
}

\author{
Brian Anderson ${ }^{1 \mathrm{I}}$, Shyamsunder Sabat ${ }^{2 \mathrm{G}}$, Amit Agarwal ${ }^{2 \mathrm{~B}}$, \\ Krishnamoorthy Thamburaj2 ${ }^{2 \mathrm{~B}}$ \\ 1 Department of Neurosurgery, Penn State University, Hershey, PA, U.S.A. \\ 2 Department of Radiology, Penn State University, Hershey, PA, U.S.A.
}

Author's address: Amit Agarwal, Department of Radiology, Penn State University, Hershey, PA, U.S.A., e-mail: amitmamc@gmail.com

\begin{abstract}
Background:
Aneurysmal rupture accounts for the majority of nontraumatic subarachnoid hemorrhage (SAH). Increasingly recognized is the occurrence of nontraumatic convexity SAH unaccounted for by aneurysmal rupture.

Case Report:

These presentations require consideration of rare but clinically significant sources of SAH. We report a patient presenting with prolonged mild headaches and acute onset of seizure like activity found to have diffuse subarachnoid hemorrhage and extensive dural venous sinus thrombosis involving the superior sagittal sinus and right transverse-sigmoid sinuses.

Conclusions:

There are few reported cases of SAH secondary to dural sinus thrombosis; however most of these are convexity hemorrhage. Sinus thrombosis presenting as diffuse SAH is extremely rare, as is showcased in this report.

MeSH Keywords: $\quad$ Sinus Thrombosis, Intracranial • Subarachnoid Hemorrhage • Tomography, Spiral Computed

PDF file: $\quad$ http://www.polradiol.com/abstract/index/idArt/894122
\end{abstract}

\section{Summary}

\section{Background}

Nontraumatic, nonaneurysmal subarachnoid hemorrhage (SAH) often presents a diagnostically difficult scenario for clinicians. While a perimesencephalic hemorrhage presents with findings of hemorrhage within the basal cisterns, convexity SAH is not accounted for by this etiology. Such a presentation accounts for less than 10\% of SAHs with cerebral amyloid angiopathy constituting nearly half of such cases. The remainders are comprised of rare etiologies including cerebral venous sinus thrombosis, reversible cerebral vasoconstriction syndrome, posterior reversible encephalopathy syndrome, and a mixture of other rarely reported cases [1,2]. Although the pathophysiological abnormalities leading to SAH in these cases are not completely understood, improved imaging is providing increased identification of such cases and our understanding is improving. We reported a case of a patient presenting with insidious headaches over several months and acute left-sided jerking movement consistent with seizure activity. Imaging identified extensive dural venous sinus thrombosis with associated diffuse SAH.

\section{Case Report}

A 42-year-old male presented to our institution following several episodes of uncontrolled jerking movements and dysesthesia of the left extremities. The final episode resulted in the loss of consciousness and prompted him to present for evaluation. There was no witnessed trauma and no external indications of injury. His only significant medical history included diabetes and hypertension, both poorly controlled at the time of presentation, and active tobacco use. Family history was significant for a mother with pulmonary embolus and associated deep vein thrombosis. Neurologic examination was non-focal with appropriate orientation, speech, and motor findings.

A head CT was obtained which revealed diffuse SAH, most prominent along the frontotemporal convexities (Figure 1A, 1B). The patient was admitted to the neuroscience intensive care unit and evaluation for aneurysmal rupture was continued. CT angiogram was subsequently performed which did not reveal any intracranial aneurysm. Brain MRI and MR venogram were carried out 


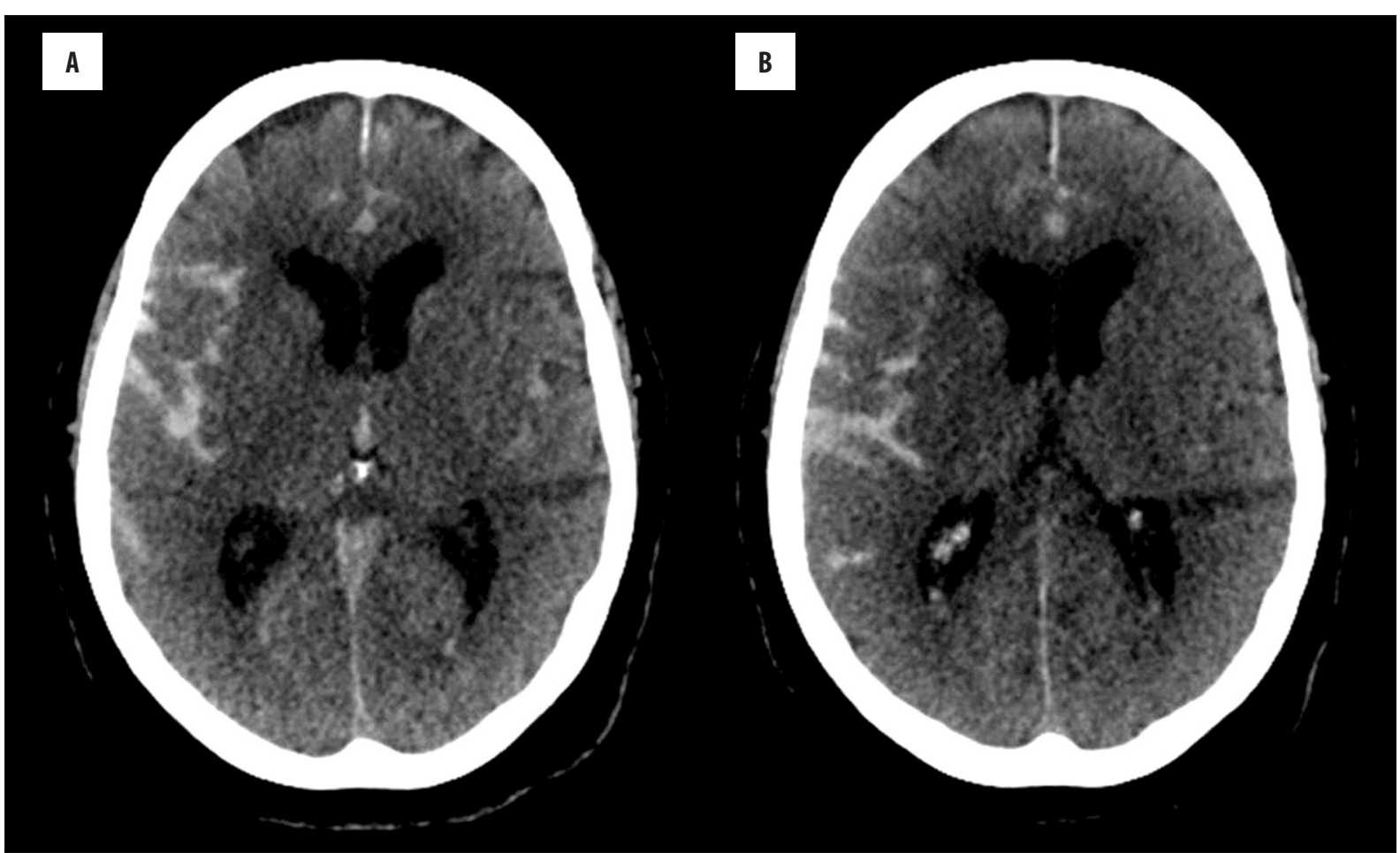

Figure 1. (A, B) Two contiguous axial head CT images showing diffuse subarachnoid hemorrhage, most prominent along the right fronto-temporal convexity. No parenchymal changes seen.

and demonstrated dense dural venous sinus thrombosis involving the posterior two-thirds of the superior sagittal sinus, as well as the right transverse and sigmoid sinuses [Figure 2A-2D]. That appeared as an intrinsic bright signal on Tl-weighted images with complete non-visualization of those sinuses on the MR venogram. No restricted diffusion was noted to suggest infarct. No parenchymal signal changes were seen.

Intravenous heparin was initiated and transition to Coumadin therapy was made. The patient was given Keppra for presumed seizure activity without additional events. A complete hypercoagulation evaluation was completed, including fibrinogen, protein C and S, antithrombin III, plasminogen activity, lupus anticoagulant, and dilute Russell's viper venom without abnormal findings. The anticardiolipin panel and B2 glycoprotein panel were within normal limits. The patient was discharged with appropriate anticoagulation and appropriate follow-up. A repeat MRI at two weeks found stable sinus thrombosis with resolution of subarachnoid hemorrhage. A follow-up MRI at 4 months showed partial recanalization of the dural sinuses. Regular outpatient follow-ups find continued symptom resolution.

\section{Discussion}

Atypical presentation of SAH without evidence of trauma or aneurysmal rupture is a rare finding requiring careful consideration of uncommon pathologies. While the number of reported cases of cerebral venous sinus thrombosis (CVST) presenting as SAH is increasing, a careful reminder of this unusual presentation is warranted [3-7]. Significant clinical variation exists in patients who present with CVST which can often delay the diagnosis [8]. When SAH is identified, thorough imaging must be completed to ensure the bleed is from a nonaneurysmal source. Unusual but recognized distal aneurysms and dural arteriovenous fistulas (DAVF) may present an immediate and significant mortality risk [9]. Cortical venous drainage of DAVF may not only result in SAH but also be the causative agent in the formation of CVST [10]. Digital subtraction angiography (DSA) is considered the gold standard for vascular evaluation in the setting of SAH but may not be available at all centers. Evaluation of contrast flow in the venous phase is likely to suggest the presence of CVST whether or not sinus thrombosis is suspected [10]. The non-invasive imaging may stand as an alternative to DSA requiring appropriate venous phase sequencing be ordered specifically. If arterial etiologies are ruled out, venography must be considered to asses the presence of venous thrombosis.

The specific pathogenesis of SAH as a result of CVST is not fully understood. Some consideration is given to elevated cortical venous pressures resulting in a rupture of the fragile and inadequate vein wall releasing blood into the subarachnoid space [10]. Less focal vascular changes are suspected by some, based on the presence of blood in CSF even in patients without imaging significant for SAH [11-13]. Considering the relatively minor radiographic and diagnostic findings as well as the unreliable clinical presentation, CVST must be actively considered within the differential to avoid delayed diagnosis.

Once identification of CVST is made, immediate initiation of anticoagulation therapy is critical $[8,14,15]$. With medical therapy success in the vast majority of patients, more invasive techniques are reserved for patients who fail 


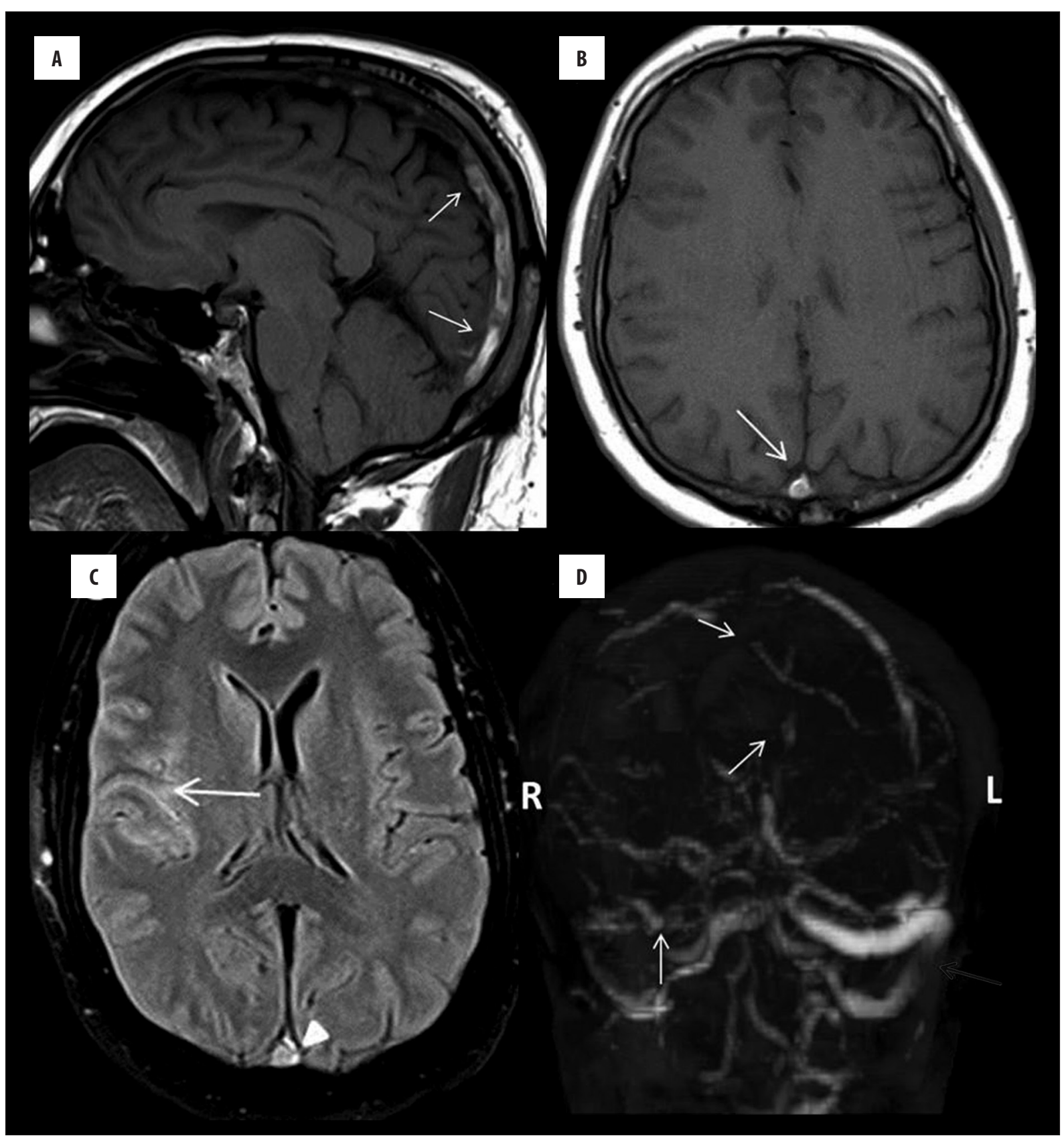

Figure 2. Sagittal and axial T1-weighted images (A, B) showing an intrinsic bright signal within the superior sagittal sinus (arrows) with loss of a normal flow void. Axial FLAIR image $(\mathbf{C})$ in the same patient showing a bright signal within the sulci (arrow) corresponding to the SAH seen on (T, along with a bright thrombus signal within the superior sagittal sinus (arrowhead). Complete non-opacification of the superior sagittal, right transverse and sigmoid sinuses (white arrows) on MR venogram (D). The left transverse sinus is patent (grey arrow).

conservative measures or experience significant complications. Although uncommon, recognition of potential elevation of intracranial pressure must not be overlooked. For patients with an unreliable neurological status, intracranial monitoring should be considered $[8,16]$. Non-invasive monitoring via transcranial Doppler ultrasonography is available for consideration [17]. Invasive endovascular techniques including intravenous thrombolysis and mechanical thrombectomy are reported. With high periprocedural complications and unproven outcome improvement, these techniques are typically reserved for truly refractory cases [18-21].

\section{Conclusions}

Nontraumatic, nonaneurysmal causes of SAH remain a rare, poorly understood phenomenon. A careful exclusion of arterial pathogenesis followed by appropriate venous sources may occasionally identify CVST as the causative source. Subsequent venous congestion may lead to SAH with variable clinical presentation which may be difficult to identify. Recognition of this uncommon etiology is essential to early diagnosis and treatment pivotal in avoiding the potentially life-threatening complications of progressive cerebral venous sinus thrombosis. 


\section{References:}

1. Carvi y Nievas MN, Archavlis E: Atypical causes of nontraumatic intracranial subarachnoid hemorrhage. Clin Neurol Neurosurg, 2009; $111(4)$ : 354-58

2. Khurram AF, Kleinig TP, Leyden JF: Clinical associations and causes of convexity subarachnoid hemorrhage. Stroke, 2014; 45(4): 1151-53

3. Rice H, Tang YM: Acute subarachnoid haemorrhage: a rare presentation of cerebral dural sinus thrombosis. Australas Radiol, 2006; 50(3): 241-45

4. Pradhan S, Yadav R, Diwakar H, Phadke RV: Subarachnoid hemorrhage following chronic dural venous sinus thrombosis. Angiology, 2007; 58(4): 498-501

5. Kurosu A, Suzukawa K, Amo M et al: Perimesencephalic nonaneurysmal subarachnoid hemorrhage caused by cavernous sinus thrombosis: case report. Neurol Med Chir (Tokyo), 2007; 47(6): 258-60

6. Panda S, Prashantha DK, Shankar SR, Nagaraja D: Localized convexity subarachnoid haemorrhage - a sign of early cerebral venous sinus thrombosis. Eur J Neurol, 2010; 17(10): 1249-58

7. Sharma B, Satija V, Dubey P, Panagariya A: Subarachnoid hemorrhage with transient ischemic attack: another masquerader in cerebral venous thrombosis. Indian J Med Sci, 2010; 64(2): 85-89

8. Saposnik G, Barinagarrementeria F, Brown RD et al: Diagnosis and management of cerebral venous thrombosis: a statement for healthcare professionals from the American Heart Association/ American Stroke Association. Stroke, 2011; 42(4): 1158-92

9. Biondi A, Casasco A, Houdart E et al: [Evolution of angiographic signs of venous hypertension and clinical signs of intracranial hypertension in intracranial dural arteriovenous fistulas]. $\mathrm{J}$ Neuroradiol, 1999; 26(1): 49-58 [in French]

10. Oppenheim C, Domigo V, Gauvrit JY et al: Meder JF. Subarachnoid hemorrhage as the initial presentation of dural sinus thrombosis. Am J Neuroradiol, 2005; 26(3): 614-17
11. Al-Hashel JY, John JK, Vembu P: Venous thrombosis of the brain. Retrospective review of 110 patients in Kuwait. Neurosciences (Riyadh), 2014; 19(2): 111-17

12. Park JH, Yoon SH: New concept of cerebrospinal fluid dynamics in cerebral venous sinus thrombosis. Med Hypotheses, 2008; 70(1): $143-47$

13. Bousser MG, Chiras J, Bories J, Castaigne P: Cerebral venous thrombosis - a review of 38 cases. Stroke, 1985; 16(2): 199-213

14. Hegazi MO, Ahmed S, Sakr MG, Hassanien OA: Anticoagulation for cerebral venous thrombosis with subarachnoid hemorrhage: a case report. Med Princ Pract, 2010; 19(1): 73-75

15. Viegas LD, Stolz E, Canhão P, Ferro JM: Systemic thrombolysis for cerebral venous and dural sinus thrombosis: a systematic review. Cerebrovasc Dis, 2014; 37(1): 43-50

16. Hanley DF, Feldman E, Borel CO et al: Treatment of sagittal sinus thrombosis associated with cerebral hemorrhage and intracranial hypertension. Stroke, 1988; 19(7): 903-9

17. Wakerley B, Yohana $\mathrm{K}$, Luen Teoh $\mathrm{H}$ et al: Non-invasive intracranial pressure monitoring with transcranial Doppler in a patient with progressive cerebral venous sinus thrombosis. J Neuroimaging, 2014 24(3): 302-4

18. Siddiqui FM, Banerjee C, Zuurbier SM et al: Mechanical thrombectomy versus intrasinus thrombolysis for cerebral venous sinus thrombosis: a non-randomized comparison. Interv Neuroradiol, 2014; 20(3): 336-44

19. Choudhri O, Feroze A, Marks MP, Do HM: Endovascular management of cerebral venous sinus thrombosis. Neurosurg Focus, 2014; 37(V1Supplement): 1

20. Coutinho JM, Middeldorp S, Stam J: Advances in the treatment of cerebral venous thrombosis. Curr Treat Options Neurol, 2014; 16(7): 299

21. Guo X, Guan S, Fan Y, Song L: Local thrombolysis for severe cerebral venous sinus thrombosis. Am J Neuroradiol, 2012; 33(6): 1187-90 